\title{
Seizure as the Rare Manifestation in a Young Healthy Adult with Pulmonary Embolism: Case Report
}

\author{
Dr.Kamal Preet Palta ${ }^{1}$, Dr.Sarmad Pandith ${ }^{2}$ \\ Department Of Emergency Medicine ,Max Smart Superspeciality Hospital ,New Delhi,India
}

\begin{abstract}
A 31 year old healthy young male with sudden syncope and witnessed seizure activity was discovered to have massive pulmonary embolism. Cardiac ECHO also confirmed elevated right ventricular pressure. This literature is based on unusual presentation of seizure as the initial manifestation of pulmonary embolism. Clinicians need to be more aware that pulmonary embolism is important to consider within the differential diagnosis for unexplained new onset of seizure activity.
\end{abstract}

\section{Introduction}

New onset of unexplained seizure activity in an adult with no structural lesions and a negative family history for seizure requires detailed review of the history and all possible explanations need to be carefully considered. A remarkable case illustrating this concept is provided here, where a previous healthy young adult was found to have massive pulmonary embolism with seizure activity as the presenting sign. A cardiac ECHO demonstrating elevated right ventricular pressure led to the diagnosis of pulmonary embolism.

\section{Case Report}

A 31 year old male patient was referred to Emergency from Neurological clinic on $4^{\text {th }}$ January 2017 with an episode of syncope and tonic clonic seizure. According to the patients attendant he had been complaining of left lower leg pain for past 3 days and since morning he had shortness of breath followed by a witnessed episode of seizure first time in his life. The patient was diagnosed hypothyroid and was taking Tablet Thyroxin. Family history was negative for any seizure disorder.

On arrival to Emergency, patient was disoriented and confused. He was profusely sweating. There was evidence of respiratory distress but no sign of cyanosis. Patient was Afebrile and Blood Pressure was not recordable with a regular heart rate of 155 beats per minute with no gallop and murmur. Oxygen saturation was $86 \%$ on room air. Physical Examination revealed feeble rapid pulse but pulse in the left lower extremity was not palpable. There was no organomegaly and no palpable mass.

Initial resuscitation was started and patient was put on 10 liters Oxygen through facial mask and Intravenous fluid. Injection Levetiracetam $1000 \mathrm{mg}$ was also given.

Patient's vitals were still deteriorating after initial resuscitation and was haemodynamically unstable, so patient was immediately intubated and put on ventilator. C.T Head as prescribed by neurologist and C.T Pulmonary angiography prescribed by cardiologist was not possible. So ECG , Echocardiography and Ultrasonography Venous Doppler of Lower Limb was immediately done in emergency.

- ECG showed Sinus Tachycardia with S1Q3T3 pattern.

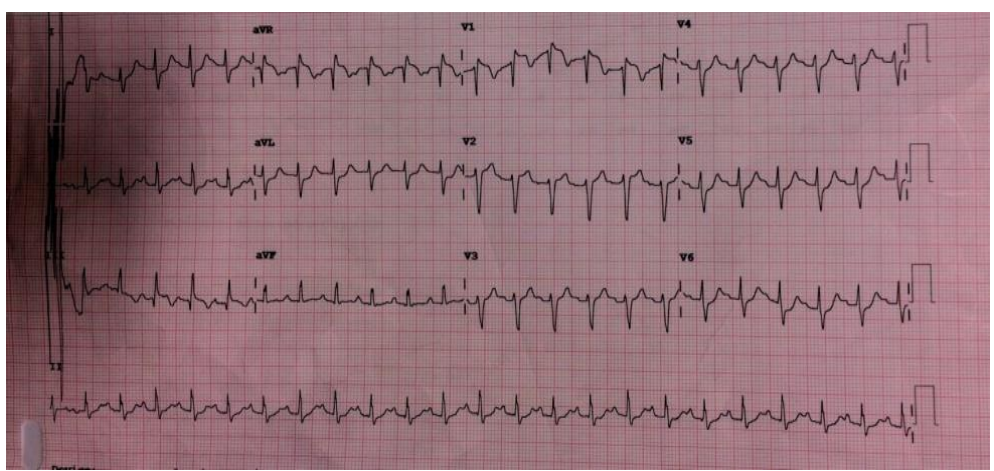

- Cardiac ECHO was done which revealed severely dilated right ventricle, measuring $70 \mathrm{~mm}$, with severely reduced systolic function.

- USG Venous Doppler of Left Lower Limb revealed A short segment in the distal most part of left CFV and adjacent left SFV appears partially compressed. 


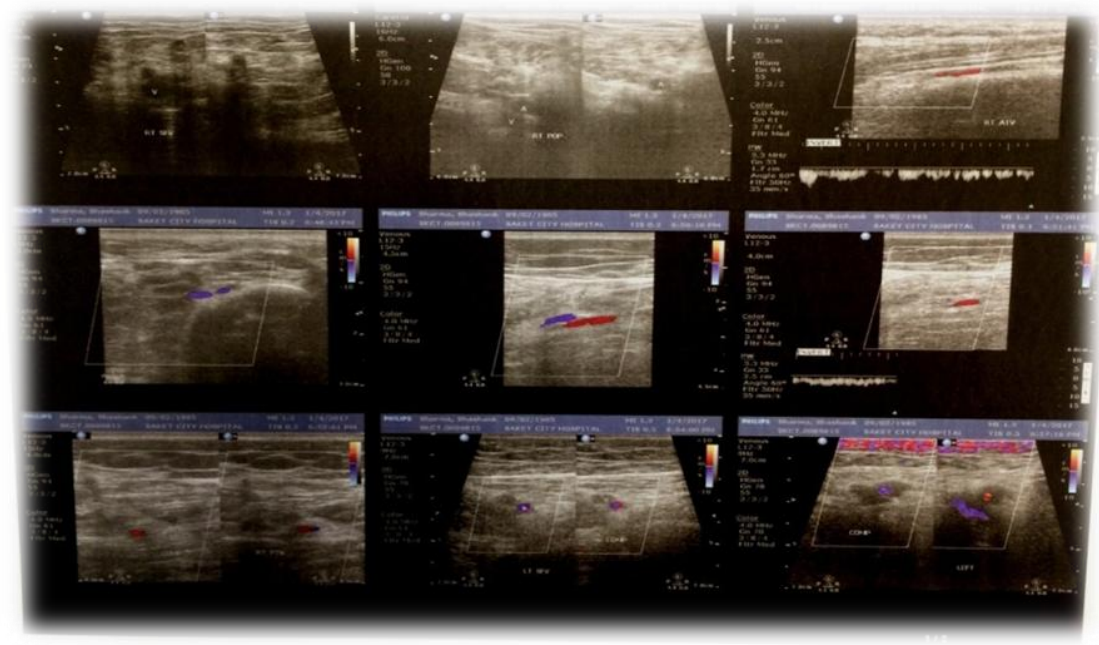

After confirming the diagnosis and within half an hour of his arrival to the emergency room, 100mg of intravenous Alteplase as slow intravenous infusion over 2 hours was immediately started. He was later started on intravenous heparin. Significant improvement was observed after thrombolytic therapy.

Patient was shifted to CCU and was continuously monitored for 12 hours. The patient significantly improved and was eventually taken off from ventilator support. The patient became haemodynamically stable with normal respiratory rate and maintaining oxygen saturations at room air. No episode of seizure was witnessed.

Repeat ECG was done which showed normal rhythm.

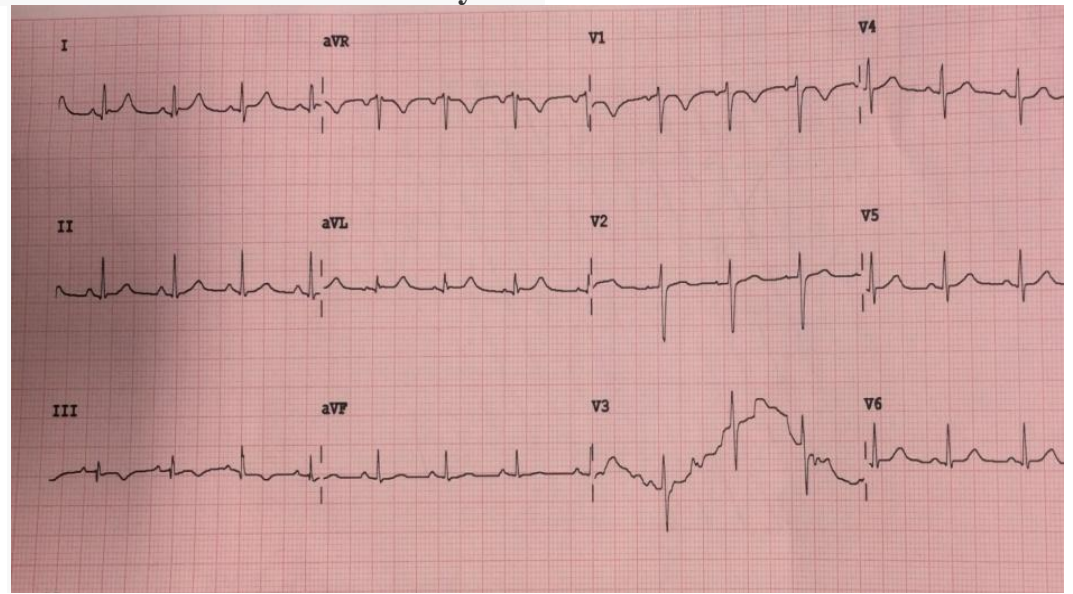

Repeat echocardiogram showed normal right ventricular size and systolic function.

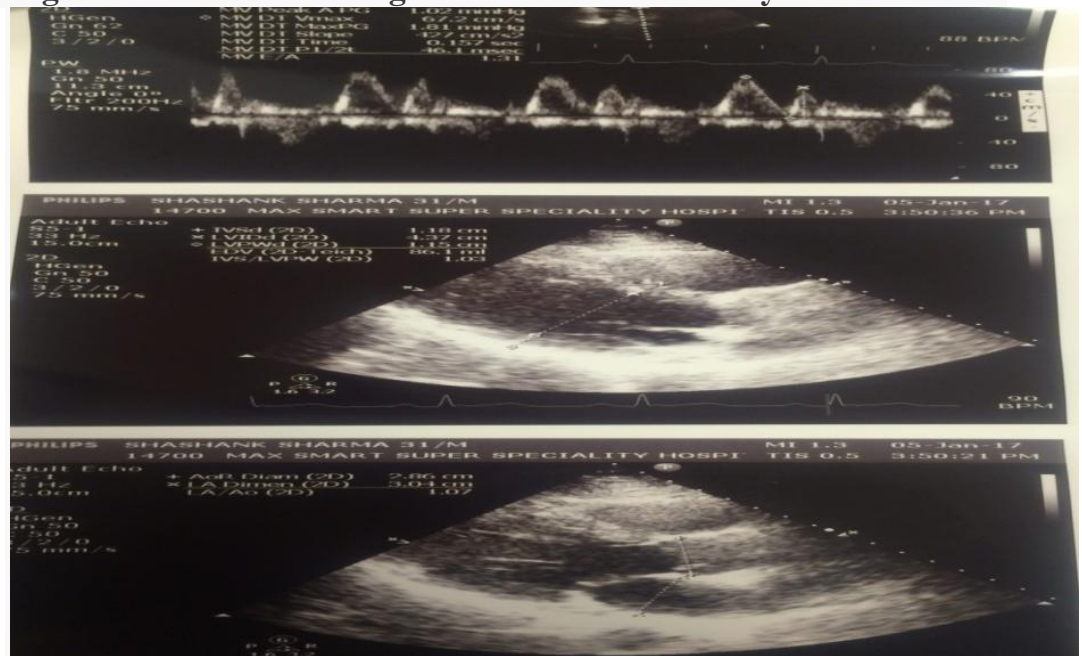




\section{Discussion}

This case very likely represented a rare presentation for pulmonary embolism (PE) as a sudden syncope with generalized tonic-clonic seizure activity. A detailed review of the literature revealed that this has been encountered by other clinicians, where autopsy confirmation was made for PE. The literature on this topic is well reviewed within the case report by Fred and Yang, who described sudden syncope and generalized seizure activity in a previously healthy 37-year old. The pathophysiology behind seizure as the presenting sign for massive PE is well described by Marine and Goldhaber, who reported two cases and felt that massive PE caused transient right ventricular failure, and decreased cardiac output, causing transient global cerebral hypoperfusion.

\section{Conclusion}

In summary, the astute clinician needs to be aware of all cardiovascular possibilities including arrhythmia as explanations for sudden syncope with unexplained new onset of generalized seizure activity. However, as shown by this case, life threatening pulmonary embolism is clearly within the differential diagnosis list even in young patients without risk factors. When considering new onset of unexplained generalized seizure activity in an adult, the neurologist should always consider the possibility of life threatening PE, despite the lack any obvious risk factors.

\section{Learning Point}

CT Pulmonary angiography is the diagnostic test for pulmonary embolism but in case of scenario where patient is heamodynamically unstable and interventions has to be started immediately Echocardiography and Ultrasonography can be used as the important tools for diagnosis of pulmonary embolism in emergency. So this case report emphasizes the importance of use of Echocardiography and Ultrasonography in Emergency.

\section{References}

[1]. Fred HL, Yang M. Sudden loss of consciousness, dyspnea, and hypoxemia in a previously healthy young man. Circulation 1995;15(91):3017-9.

[2]. Marine JE, Goldhaber SZ. Pulmonary embolism presenting as seizures. Chest 1997;112:840-2. 